\title{
Regression modeling and neural computing for predicting the ultimate tensile strength of friction stir welded aerospace aluminium alloy
}

\author{
Akshansh MISHRA, Jonathan Ve VANCE
}

DOI: 10.30464/jmee.2019.3.3.221

Cite this article as:

Mishra A., Vance J. V.. Regression modeling and neural computing for predicting the ultimate tensile strength of friction stir welded aerospace aluminium alloy. Journal of Mechanical and Energy Engineering, Vol. 3(43), No. 3, 2019, pp. 221-226.

\section{VOLUME 3(43) | No. 3 | SEPTEMBER 2019}

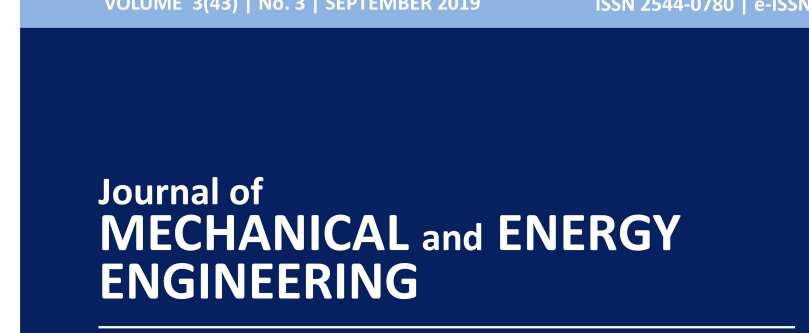

Editor-in-Chief

Waldemar Kuczyński

Editors

Krzysztof Rokosz | Krzysztof Nadolny
Journal of Mechanical and Energy

Engineering

Website: jmee.tu.koszalin.pl

ISSN (Print): 2544-0780

ISSN (Online): 2544-1671

Volume: 3(43)

Number: 3

Year: 2019

Pages: 221-226

Article Info:

Received 1 August 2019

Accepted 21 October 2019

\section{Open Access}

This article is distributed under the terms of the Creative Commons Attribution 4.0 (CC BY 4.0) International License (http://creativecommons.org/licenses/by/4.0/), which permits unrestricted use, distribution, and reproduction in any medium, provided you give appropriate credit to the original author(s) and the source, provide a link to the Creative Commons license, and indicate if changes were made. 


\title{
REGRESSION MODELING AND NEURAL COMPUTING FOR PREDICTING THE ULTIMATE TENSILE STRENGTH OF FRICTION STIR WELDED AEROSPACE ALUMINIUM ALLOY
}

\author{
Akshansh MISHRA $^{1 *}$, Jonathan Ve VANCE ${ }^{2}$ \\ ${ }^{1 *}$ Center for Computational Intelligence Friction Stir Welding, Stir Research Technologies, \\ Uttar Pradesh, India, e-mail: akshansh.frictionwelding@gmail.com \\ ${ }^{2}$ Department of Engineering Design, Indian Institute of Technology Madras, Ćennaj, Tamilnadu, India
}

(Received 1 August 2019, Accepted 21 October 2019)

\begin{abstract}
AA7075 is an aluminum alloy which is almost as strong as steel, yet it weighs just one third as much. Unfortunately its use has been limited, due to the fact that pieces of it could not be securely welded together by the traditional welding process. Friction Stir Welding (FSW) process overcomes the limitations of conventional welding process. In our present work we have used Artificial Neural Network which is Artificial Intelligence based technique used for prediction purpose. The main objective of our present work is to compare the predicted results of the Ultimate Tensile Strength (UTS) of Friction Stir welded similar joints through Regression modeling and Artificial Neural Network (ANN) modeling. It was observed that the linear regression algorithm is able to make more accurate predictions compared to neural network algorithm for small dataset.
\end{abstract}

Keywords: artificial neural network, regression model, friction stir welding

\section{INTRODUCTION}

The manufacturing industries have faced many challenges in cutting cost and maintaining efficiency in while developing welding based product. Welding is a very expensive manufacturing process and some of the challenges that are faced while carrying out this process are the factors that amount to porosity, undercut, deformation, cracks, incomplete penetration and fusion.

To handle this problem an effective manufacturing process has been developed, called as Friction Stir Welding (FSW). The main advantage of Friction Stir Welding over other conventional welding process is that it doesn't melt the base metal which will further not lead to the defects formation arises due to entrapment of the gas in the weld zone. Friction Stir Welding is a solid state joining method which plasticize the base metal to be joined by the heat generation due to friction generated by the contact surfaces of the tool and the base metal. If we talk technically, Friction Stir Welding is governed by the parameters like the tool rotational speed, traverse speed, tool tilt angle and various geometrical aspects of the tool used during the process [1]. The major advantage that this technique has over the other conventional techniques is the ability to join the alloys which are difficult to join by conventional welding process like Tungsten Inert Gas Welding and Metal Inert Gas welding. High temperature alloys like Titanium can be easily welded by Friction Stir Welding process. Also, lesser welding time and inducing lower heat energy meaning better efficiency are other edges that Friction Stir Welding has over its competitions.

Now the rising issues in the manufacturing industry is that a welding process needs to be expeditious and accurate to improve performance and strength of the welding structure. Hence an optimization study and test of Friction Stir Welding process needs to be carried out in order to improve the industry standards. However, this optimization approach requires to measure extremely sensitive welding factors which is difficult to carry out with 
human intervention. So one of the most best method is to use machine deep learning approach. The deep learning techniques which can be used in FSW research domain are Artificial Neural Networks (ANNs) and Machine Vision system for determining the quality of the joints.

Artificial Neural Network is primarily designed to replicate information processing of the human brain or the neural system of a human body. Neural network consists of basic elements called neurons which process the input signal from a defined system and push it to a transfer function which is differentiable so as to generate an output which works in a similar way as the brain neurons. The main question that arises now, is that why to use this system? A very logical and practical explanation would be due to the problems arising in the data management systems built by our predecessors which is incapable of handling the technological advancement and growth in the industry. The neural network can easily overcome challenges like the input data size and the incoherency of the unstructured data that is available in abundance. The neural network works on the principle of training functions that update bias values and weights, so as to reduce probability of errors. When talking about the Architecture of the neural network, it is a multi-layer network consisting of input parameter feed, output layer, and at least one training function to processes input to yield output as shown in the Figure 1.

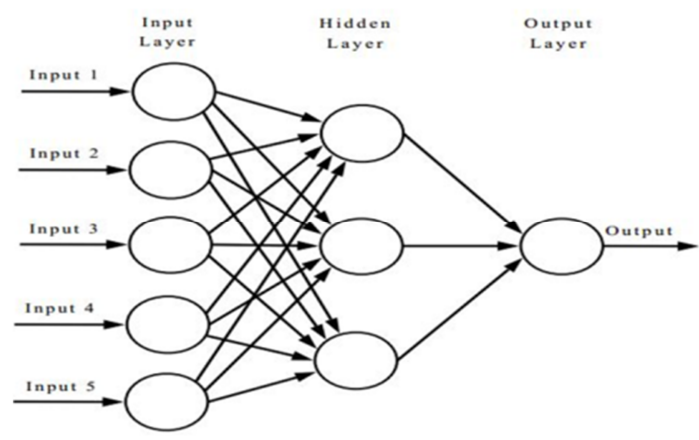

Fig. 1. Schematic representation of Neural Network Architecture

Observe the Figure 2 in order to understand the mechanism of the working of Artificial Neural Network.

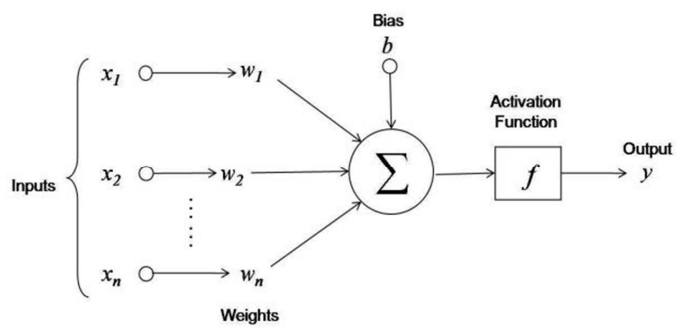

Fig. 2. Block diagram of Neural Network Architecture
The datasets extracted from global sources are provided in the form of input layer, which then propagates through hidden layers where it undergoes the training function of the output. Training holds the foremost importance in the accuracy of the prediction of response. The accuracy of the network is measured by the Mean sum of Squared Error (MSE) between the measured and predicted values. The newly adjusted weight and bias value is again sent backwards for further training. Thus this continuous iterative process of training the dataset goes on through the feedback system and will stop once an acceptable error is reached.

Friction Stir Welding (FSW) process utilizes the Artificial Neural Network Technique to reduce the number of experiments and thus it also reduced the cost of the experiment. Akshansh et al. [2] looked into the use of ANNs in the investigation of tool parameters (as well as mechanical properties of the material), for the evaluation of feedback forces which is provided by Friction Stir Welding process. It was concluded that ANNs can be used to effectively reduce cost and time, by considering the accuracy of the predictions, and that a good correlation can be observed between the predicted data obtained from the ANN and the FEM models. Chiteka et al. [3] aimed at determining the most crucial parameters that the UTS of the material depends upon (i.e., rotational speed and welding speed), and use them to predict the UTS. It talked about the complex non-linear correlation between UTS and the input parameters, which made ANNs the obvious choice of model. It was concluded that prediction of UTS using the input parameters was accurate within acceptable errors. But the reverse, i.e., prediction of input parameters from UTS was not feasible, as different combinations of input parameters produce the same UTS, and also the range of input parameters the machine can produce is also restricted. Ghetiya et al. [4] hypothesised the use of a standard 4-8-1 ANN to predict UTS from input parameters like tool shoulder diameter, tool rotational speed, welding speed and axial force. All input and output data was normalised to within a range of 0.1 to 0.9 , before training. The results of prediction were very close to experiment results. The overall $\mathrm{R}$ value for training, validation and testing was found to be bigger than 0.99 and the error was less than $3 \%$. Gupta et al. [5] predicted the values of tensile strength, average microhardness at weld nugget zone (WNZ) and average grain size at WNZ by ANN modeling and regression modeling. It was observed that the overall average percentage prediction error of ANN model was small as compared to regression model.

In our study we have trained our prediction model on Backward Propagation (BP) algorithm and we have used Rectified Linear Unit (ReLu) as an activation function for obtaining the output. In the Neural Network architecture the inputs can be tool rotational 
speed, traverse speed, tool tilt angle and geometrical parameters like tool pin diameter and too shoulder diameter. The output in the ANN architecture can be Ultimate Tensile Strength, hardness and grain size of the Friction Stir Welded joints.

\section{MATERIALS AND METHODS}

The setup for Friction Stir Welding process requires the alloys to be joined, tool which is harder than the base alloys and the fixture which is used to hold the alloy workpiece in contact throughout the joining process. AA 7075 Aluminium alloys of the dimension $250 \times 100 \times 5 \mathrm{~mm}$ is used in this study as a base alloy. The chemical composition of AA 7075 is shown in the Table 1. H13 is used as a tool material for joining the workpiece alloys. The direction of the welding was normal to the rolling direction. The tensile testing was carried out on Universal Testing Machine (UTM). Result of the tensile testing is tabulated in the Table 2.

Tab. 1. Chemical composition of AA 7075 alloy

\begin{tabular}{cc}
\hline Component & Weight \% \\
\hline $\mathrm{Al}$ & $87.1-91.4$ \\
$\mathrm{Cr}$ & $0.18-0.28$ \\
$\mathrm{Cu}$ & $1.2-2$ \\
$\mathrm{Fe}$ & $\mathrm{Max} 0.5$ \\
$\mathrm{Mg}$ & $2.1-2.9$ \\
$\mathrm{Mn}$ & $\operatorname{Max} 0.3$ \\
$\mathrm{Si}$ & Max 0.4 \\
$\mathrm{Ti}$ & Max 0.2 \\
$\mathrm{Zn}$ & $5.1-6.1$ \\
\hline
\end{tabular}

Tab. 2. Experimental Dataset

\begin{tabular}{|c|c|c|c|c|c|c|c|c|c|}
\hline \multirow{2}{*}{$\begin{array}{l}\text { Factors } \\
\text { Symbol } \\
\text { Sample nos. }\end{array}$} & \multicolumn{4}{|c|}{ Uncoded welding parameters } & \multicolumn{4}{|c|}{ Coded welding parameters } & \multirow{2}{*}{$\begin{array}{l}\text { UTS } \\
\sigma_{\mathrm{us}} \\
(\mathrm{MPa})\end{array}$} \\
\hline & $\begin{array}{l}\text { Rotation } \\
\text { speed } \\
R \\
\text { (rpm) }\end{array}$ & $\begin{array}{l}\text { Welding } \\
\text { speed } \\
W \\
(\mathrm{~mm} / \mathrm{min})\end{array}$ & $\begin{array}{l}\text { Shoulder } \\
\text { diameter } \\
D \\
(\mathrm{~mm})\end{array}$ & $\begin{array}{l}\text { Pin } \\
\text { diameter } \\
P \\
(\mathrm{~mm}) \\
\end{array}$ & $\begin{array}{l}\text { Rotation } \\
\text { speed } \\
\text { A }\end{array}$ & $\begin{array}{l}\text { Welding } \\
\text { speed } \\
\text { B }\end{array}$ & $\begin{array}{l}\text { Shoulder } \\
\text { diameter } \\
\text { C }\end{array}$ & $\begin{array}{l}\text { Pin } \\
\text { diameter } \\
\text { D }\end{array}$ & \\
\hline 1 & 425 & 50 & 13.5 & 4.5 & -1 & -1 & -1 & -1 & 465 \\
\hline 2 & 575 & 50 & 13.5 & 4.5 & 1 & -1 & -1 & -1 & 444 \\
\hline 3 & 425 & 80 & 13.5 & 4.5 & -1 & 1 & -1 & -1 & 460 \\
\hline 4 & 575 & 80 & 13.5 & 4.5 & 1 & 1 & -1 & -1 & 423 \\
\hline 5 & 425 & 50 & 16.5 & 4.5 & -1 & -1 & 1 & -1 & 450 \\
\hline 6 & 575 & so & 16.5 & 4.5 & 1 & -1 & 1 & -1 & 436 \\
\hline 7 & 425 & 80 & 16.5 & 4.5 & -1 & 1 & 1 & -1 & 462 \\
\hline 8 & 575 & 80 & 16.5 & 4.5 & 1 & 1 & 1 & -1 & 448 \\
\hline 9 & 425 & 50 & 13.5 & 5.5 & -1 & -1 & -1 & 1 & 448 \\
\hline 10 & 575 & 50 & 13.5 & 5.5 & 1 & -1 & -1 & 1 & 433 \\
\hline 11 & 425 & 80 & 13.5 & 5.5 & -1 & 1 & -1 & 1 & 480 \\
\hline 12 & 575 & 80 & 13.5 & 5.5 & 1 & 1 & -1 & 1 & 459.3 \\
\hline 13 & 425 & so & 16.5 & 5.5 & -1 & -1 & 1 & 1 & 428 \\
\hline 14 & 575 & 50 & 16.5 & 5.5 & 1 & -1 & 1 & 1 & 422 \\
\hline 15 & 425 & 80 & 16.5 & 5.5 & -1 & 1 & 1 & 1 & 466 \\
\hline 16 & 575 & 80 & 16.5 & 5.5 & 1 & 1 & 1 & 1 & 476 \\
\hline 17 & 350 & 65 & 15 & 5 & -2 & 0 & 0 & 0 & 440.6 \\
\hline 18 & 650 & 65 & 15 & 5 & 2 & 0 & 0 & 0 & 415 \\
\hline 19 & 500 & 35 & 15 & 5 & 0 & -2 & 0 & 0 & 458 \\
\hline 20 & 500 & 95 & 15 & 5 & 0 & 2 & 0 & 0 & 487 \\
\hline 21 & 500 & 65 & 12 & 5 & 0 & 0 & -2 & 0 & 448 \\
\hline 22 & 500 & 65 & 18 & 5 & 0 & 0 & 2 & 0 & 427 \\
\hline 23 & 500 & 65 & 15 & 4 & 0 & 0 & 0 & -2 & 458 \\
\hline 24 & 500 & 65 & 15 & 6 & 0 & 0 & 0 & 2 & 472 \\
\hline 25 & 500 & 65 & 15 & 5 & 0 & 0 & 0 & 0 & 466 \\
\hline 26 & 500 & 65 & 15 & 5 & 0 & 0 & 0 & 0 & 461.5 \\
\hline 27 & 500 & 65 & 15 & 5 & 0 & 0 & 0 & 0 & 455 \\
\hline 28 & 500 & 65 & 15 & 5 & 0 & 0 & 0 & 0 & 459.5 \\
\hline 29 & 500 & 65 & 15 & 5 & 0 & 0 & 0 & 0 & 453 \\
\hline 30 & 500 & 65 & 15 & 5 & 0 & 0 & 0 & 0 & 452 \\
\hline 31 & 500 & 65 & 15 & 5 & 0 & 0 & 0 & 0 & 458 \\
\hline 32 & 500 & 65 & 15 & 5 & 0 & 0 & 0 & 0 & 464 \\
\hline 33 & 500 & 65 & 15 & 5 & 0 & 0 & 0 & 0 & 465 \\
\hline 34 & 500 & 65 & 15 & 5 & 0 & 0 & 0 & 0 & 463 \\
\hline 35 & 500 & 65 & 15 & 5 & 0 & 0 & 0 & 0 & 457 \\
\hline 36 & 500 & 65 & 15 & 5 & 0 & 0 & 0 & 0 & 456 \\
\hline
\end{tabular}


The most significant inputs critical to the prediction of Ultimate Tensile Strength are the rotational speed of the tool (RS) and the welding speed (WS). For best training of any machine learning algorithm, all the inputs must be scaled to the same range. We have used the "MinMaxScaler" module in the Scikit-learn library, to scale both the input fields to within the range -1 to 1 .

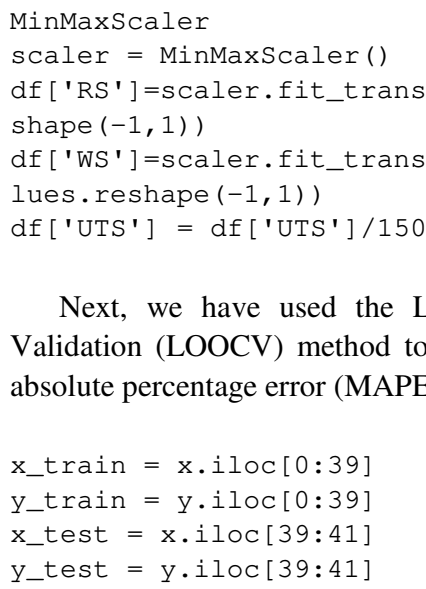

$x \_t r a i n=x$. iloc $[0: 39]$

y_train $=y \cdot$ iloc $[0: 39]$

$\mathrm{x}$ _test $=\mathrm{x}$. iloc $[39: 41]$

y_test $=y \cdot$ iloc $[39: 41]$

Next, we have used the Leave-One-Out Cross Validation (LOOCV) method to determine the mean absolute percentage error (MAPE) of the models.

This splits the input and target data frames into training and testing sets, by holding out a single input and output sample as the testing set, and the remaining as the training set; and it does this sequentially such that every sample becomes the test set exactly once. Thus, if $n$ is the number of samples, then exactly $n$ number of models are designed. The absolute errors, in percentage, on the last (test) sample in each model are averaged out to obtain the mean absolute percentage error (MAPE), which can be used to evaluate and compare different models. The two machine learning algorithms we are testing are the 2-4-8-1 neural network model as shown in the Figure 3 and the linear regression model.

The architecture we have used is a 2-4-8-1 model, with the first layer being the input layer and the last, the output layer. We have designed it using the Sequential () model from the Keras library.

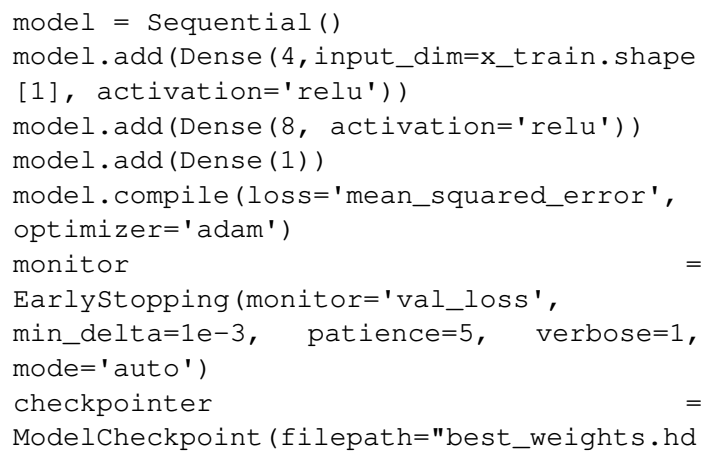

f5", verbose=0, save_best_only=True) \# save best model

model.fit (x_train,y_train, validation_data $=\left(x \_t e s t, y \_t e s t\right)$, callbacks $=$ [monitor, check pointer], verbose $=0$, epochs $=1000$ )

model.load_weights ('best_weights.hdf5') \#

load weights from best model

y_pred_NN = model.predict (x_test)

The activation functions in each layer is the rectified linear unit ( $\mathrm{ReLu})$, and the loss function, the mean squared error (MSE). The optimization algorithm is the Adam optimization algorithm, which is an extension of the standard gradient descent algorithm.

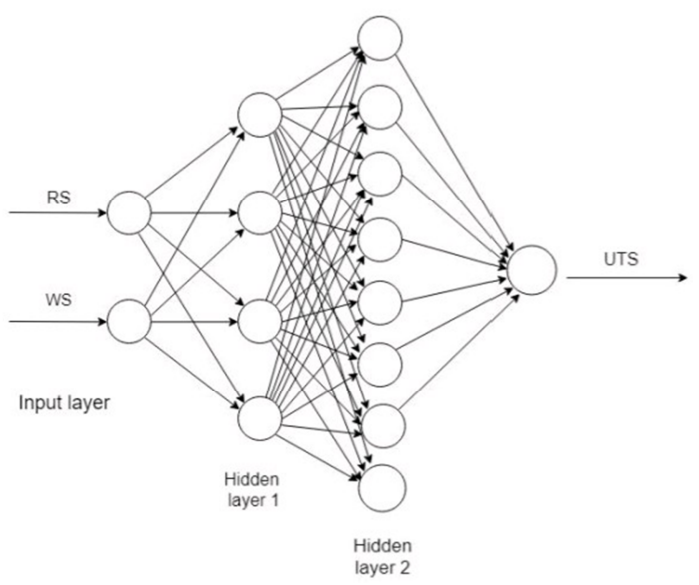

Fig. 3. Neural Network architecture used in this study

The hypothesis function for linear regression is:

$$
y=a 0+a 1 x 1+a 2 \times 2
$$

We have used the Linear Regression model from scikit-learn library. It uses the Ordinary Least Squares solver from scipy, to converge to the global minimum.

Based on the data, linear regression model seems to be able to make more accurate predictions. To test this, we have trained the neural network as well as the linear regression models on the entire dataset. Further, we have chosen 3 new samples which have not been used for training either model, and compared the predictions from both models with the experimental values.

\section{RESULTS AND DISCUSSION}

The experimental (actual) values used for testing purpose are $472 \mathrm{MPa}, 444 \mathrm{MPa}$ and 461.5 $\mathrm{MPa}$. The values obtained by prediction from linear regression model are 452.46 MPa, 436.19 $\mathrm{MPa}$ and 453.98 MPa. Similarly, the values obtained by prediction from Artificial Neural Network Model are 441.98 MPa, 445.41 MPa and 432.59 MPa. The histogram of the experimental is shown in the Figure 4. 


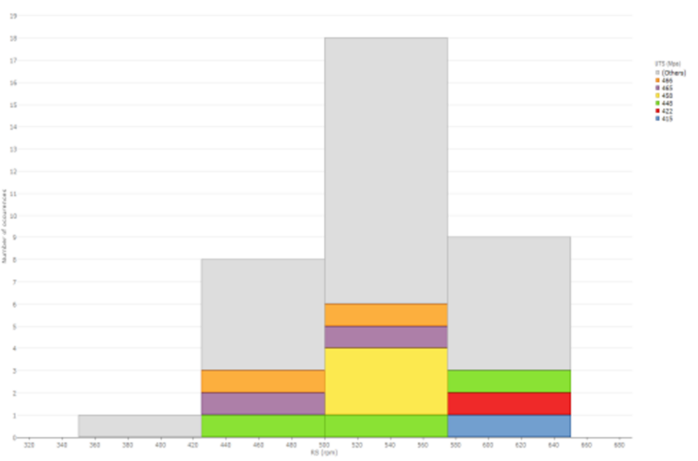

Fig. 4. Histogram of experimental dataset

Histogram of the given experimental dataset is used to see the shape of the data's distribution, especially when determining whether the output of a process is distributed approximately normally. It is observed that it is the edge peak distribution which similarly looks like the normal distribution except that it has a large peak at one tail. Usually this is caused by faulty construction of the histogram, with data lumped together into a group labeled "greater than."

The time series and frequency chart of the experimental dataset is shown in the Fig. 3 and Fig. 5.

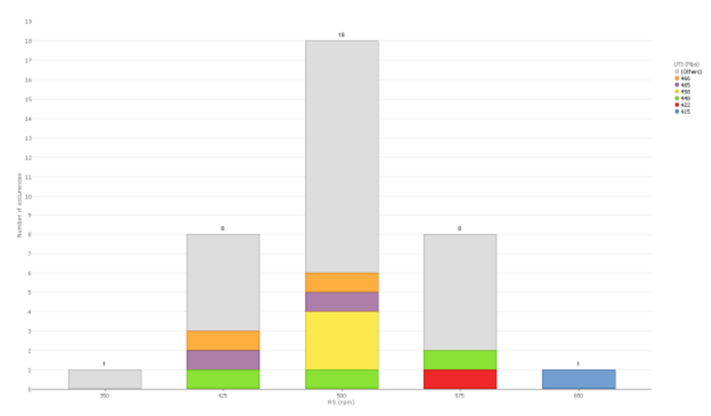

Fig. 5. Time series depiction of experimental dataset

In the Figure 5 Time Series graph illustrates data points at successive intervals of time. Each point on the chart corresponds to both a time and a quantity that is being measured. In the figure UTS is measured against Rotational Speed (rpm).

In the Figure 6 frequency table is used to display the number of occurrences of a particular value or characteristic i.e. Ultimate Tensile Strength (UTS).

Three dimensional surface plots represent threedimensional data, here there are three variables. One variable is dependent on the other two while the other two variables are independents. The surface map and contour map of the experimental dataset are show in the Figure 7 and Figure 8.

From Figure 7 it is observed that the Ultimate Tensile Strength (UTS) is a dependent variable and Rotational Speed and Welding Speed are independent variables.
Figure 8 represents the contour variation of Ultimate Tensile Strength (UTS) with the rotational speed and welding speed.

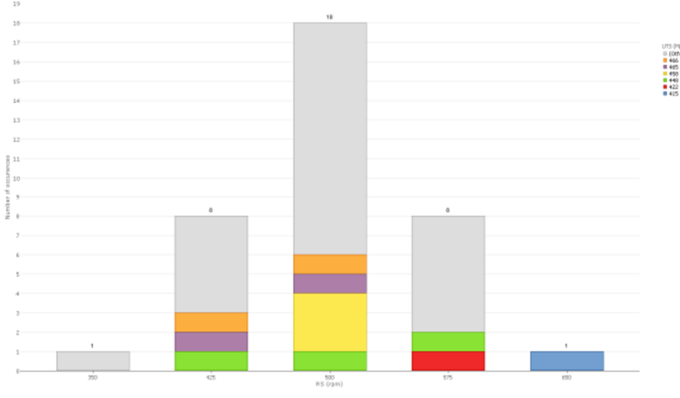

Fig. 6. Frequency chart of experimental dataset

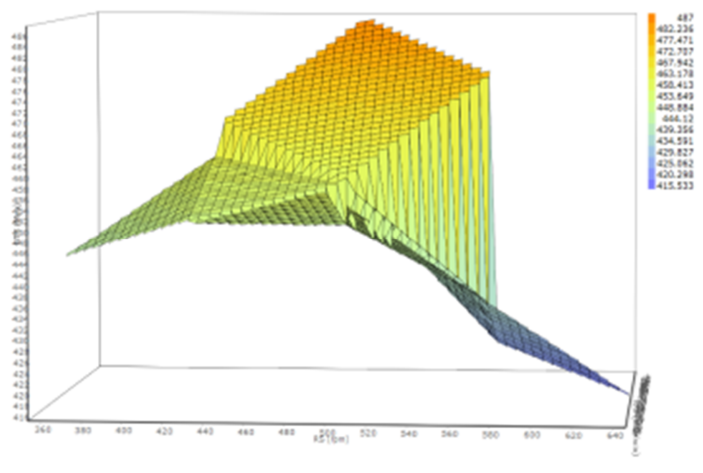

Fig. 7. Surface map of the experimental dataset

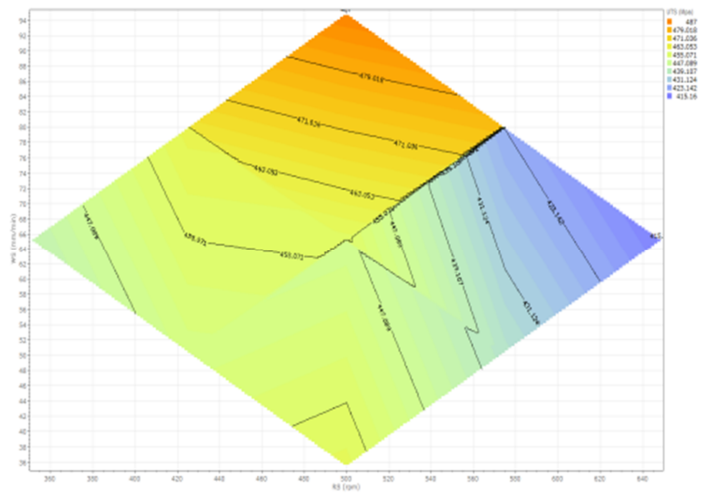

Fig. 8. Contour map of the experimental dataset

The Mean Absolute Percentage Error (MAPE) from the Leave-One-Out Cross Validation (LOOCV) method for the Artificial Neural Network model is calculated to be $7.04 \%$. From the Leave-One-Out Cross Validation method, a mean absolute percentage error (MAPE) of only $2.55 \%$ was obtained for the Regression model.

Average error $\%$ obtained for the neural network model is $4.31 \%$ while Average error \% for linear regression model is $2.52 \%$. 


\section{CONCLUSIONS}

The mean absolute percentage error (MAPE) calculated on the Leave-One-Out Cross Validation (LOOCV) method gave the following values for 2-4-8-1 neural network model and a regression analysis method:

For regression analysis, MAPE is equal to $2.55 \%$ and for 2-4-8-1 neural network model and MAPE is equal to $7.04 \%$. On running the algorithms on the new samples, we have obtained: Average error \% (neural network) is equal to $4.31 \%$ and Average error \% (linear regression) is equal to $2.52 \%$. From the mean absolute percentage error (MAPE) values as well as the tabulated data, it is clear that linear regression algorithm is able to make more accurate predictions compared to neural network algorithm.

It can be concluded that for small dataset, predicted value obtained by the Regression analysis is more accurate than the predicted value obtained by the Artificial Neural Network. The accuracy of the predicted value obtained by the Artificial Neural Network can be improved by training the Neural Network on more dataset. It can be also concluded that Artificial Intelligence based technique like Artificial Neural Network can be used Friction Stir Welding research in order to reduce the experiment time and cost. Future work is required to analyse the effect of various activation functions for obtaining the output.

\section{References}

1. Mishra, R.S. and Ma, Z.Y., 2005. Friction stir welding and processing. Materials science and engineering: $\mathrm{R}$ : reports, 50(1-2), pp.1-78.

2. Mishra, A., 2018. The Use of Artificial Neural Network in Friction Stir Welding Research. Available at SSRN 3301107. https://dx.doi.org/10.2139/ssrn.3301107

3. Chiteka, K., 2014. Artificial neural networks in tensile strength and input parameter prediction in friction stir welding.

4. Ghetiya, N.D. and Patel, K.M., 2014. Prediction of tensile strength in friction stir welded aluminium alloy using artificial neural network. Procedia Technology, 14, pp.274-281, https://doi.org/10.1016/j.protcy.2014.08.036 S. K. Gupta et al., "Comparison of ANN and Regression Modeling for Predicting the Responses of Friction Stir Welded Dissimilar AA5083-AA6063 Aluminum Alloys Joint", Applied Mechanics and Materials, Vols. 813-814, pp. 415-419, 2015, https://doi.org/10.4028/www.scientific.net/ AMM.813814.415

\section{Biographical notes}

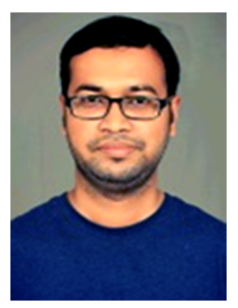

Akshansh Mishra is a founder of Stir Research Technologies which deals in collaborative research in Artificial Intelligence and Friction Stir Welding. Currently, he now works as a Principal Deep Learning Scientist in Codes \& Coffee. He had developed the first MOOC on Friction Stir Welding which is available on Udemy. His main research interests are Friction Stir Welding, Artificial Neural Network and Reinforcement Learning. He has published 7 research books dealing with Friction Stir Welding, Composites, Laser Welding and Artificial Intelligence which are available on Amazon.

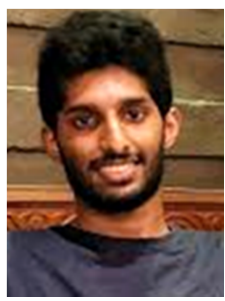

Jonathan Ve Vance is an undergraduate student in the Department of Engineering Design, IIT Madras. His main research interests are Artificial Intelligence, Neural Network and Deep Learning. 